\title{
Modulation of small GTPase activity by NME proteins
}

\author{
Vedrana Filić ${ }^{1} \cdot$ Maja Marinović ${ }^{1} \cdot$ Marko Šoštar $^{1} \cdot$ Igor Weber $^{1}$
}

Received: 20 May 2017 / Revised: 6 December 2017 / Accepted: 29 December 2017 / Published online: 12 February 2018

(c) United States \& Canadian Academy of Pathology 2018

\begin{abstract}
NME proteins are reported to influence signal transduction activity of small GTPases from the Ras superfamily by diverse mechanisms in addition to their generic NDP kinase activity, which replenishes the cytoplasmic pool of GTP. Comprehensive evidence shows that NME proteins modulate the activity of Ras GTPases, in particular members of the Rho family, via binding to their major activators GEFs. Direct interaction between several NMEs and Ras GTPases were also indicated in vitro and in vivo. These modes of regulation are mainly independent of the NME's kinase activity. NMEs also modulate the Ras-mediated signal transduction by interfering with the formation of a Ras signaling complex at the plasma membrane. In several examples, NMEs were proposed to perform the role of GAP proteins by promoting hydrolysis of the bound GTP, but this activity still requires additional verification. Early suggestions that NMEs can activate small GTPases by direct phosphorylation of the bound GDP, or by high-rate loading of GTP onto a closely apposed GTPase, were largely dismissed. In this review article, we survey and put into perspective published examples of identified and hypothetical mechanisms of Ras signaling modulation by NME proteins. We also point out involvement of NMEs in the transcriptional regulation of components of Ras GTPases-mediated signal transduction pathways, and reciprocal regulation of NME function by small GTPases, particularly related to NME's binding to membranes.
\end{abstract}

\section{Introduction}

Small GTP-hydrolases (GTPases) from the Ras superfamily are omnipresent signaling proteins that play important roles in a wide range of vital cellular processes [1,2]. Since small GTPases are active only in the GTP-bound form, their function depends on a steady supply of GTP. Small GTPases are activated by guanine nucleotide exchange factors (GEFs) that facilitate the release of GDP from inactivated, GDP-bound protein. The subsequent preferential binding of GTP to the nucleotide-binding site of a GTPase depends on a local overabundance of GTP relative to GDP [3-6]. It is, therefore, reasonable to expect that proteins believed to be involved in the maintenance of a 10fold surplus of intracellular GTP compared to GDP, such as nucleoside-diphosphate kinases from the NME family, would modulate the activity of small GTPases primarily through their nucleoside-diphosphate (NDP) kinase activity.

Igor Weber

iweber@irb.hr

1 Ruđer Bošković Institute, Division of Molecular Biology, Bijenička 54, HR-10000 Zagreb, Croatia
However, as we will outline in this short review, the interplay between NME proteins and small GTPases is far more complex.

Among 10 members of NME protein family in humans, those from the group I (NMEs 1-4) exhibit a significant NDP kinase activity and catalyze transfer of the $\gamma$ phosphate from nucleoside triphosphates (mainly ATP) to nucleoside diphosphates [7]. It was, therefore, postulated early on that the NDP kinase activity of NME proteins is pivotal in maintaining the proper balance between NTP and NDP species, including guanine nucleotides, in cellular nucleotide pools (Fig. 1a) [8]. In HeLa cells, however, the concentration of NTPs, including GTP, remained virtually unchanged upon inactivation of NME1 and NME2 by siRNA [9], indicating that the influence of NME proteins on GTPase proteins does not have to be mediated by the regulation of bulk GTP concentration, or that the amounts of NME that are still expressed after the siRNA silencing are sufficient. Experimental evidence for alternative mechanisms, such as a focused loading of GTP by the substrate channeling mechanism or direct phosphorylation of the bound GDP $[10,11]$ was either retracted $[12,13]$ or critically challenged [14-16].

As we will expound in the following, NME proteins appear to modulate the activity of Ras superfamily GTPases 
Fig. 1 Proposed mechanisms for the modulation of small GTPase activity and signaling by NME proteins. a Production of GTP by generic NDP kinase activity of NME proteins. b Inhibition of RhoGEF activity by NME proteins. $\mathbf{c}$ GAP activity of NME proteins toward small GTPases. d Direct interaction of small GTPases with NME proteins. e Inhibition of signaling downstream of Ras by NME proteins. f Direct delivery of NME-generated GTP to small GTPases (channeling). g Direct phosphorylation of GTPasebound GDP by NME proteins (phosphorylation in situ). Symbols: NME-NME protein; D-GDP; T-GTP; G-small GTPase; P-phosphate; SEQsequestrating protein; EReffector of Ras; KSR-kinase suppressor of Ras a

generic NDP kinase activity

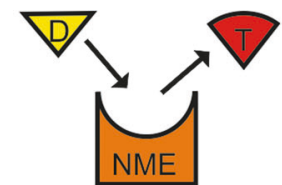

C

GAP activity
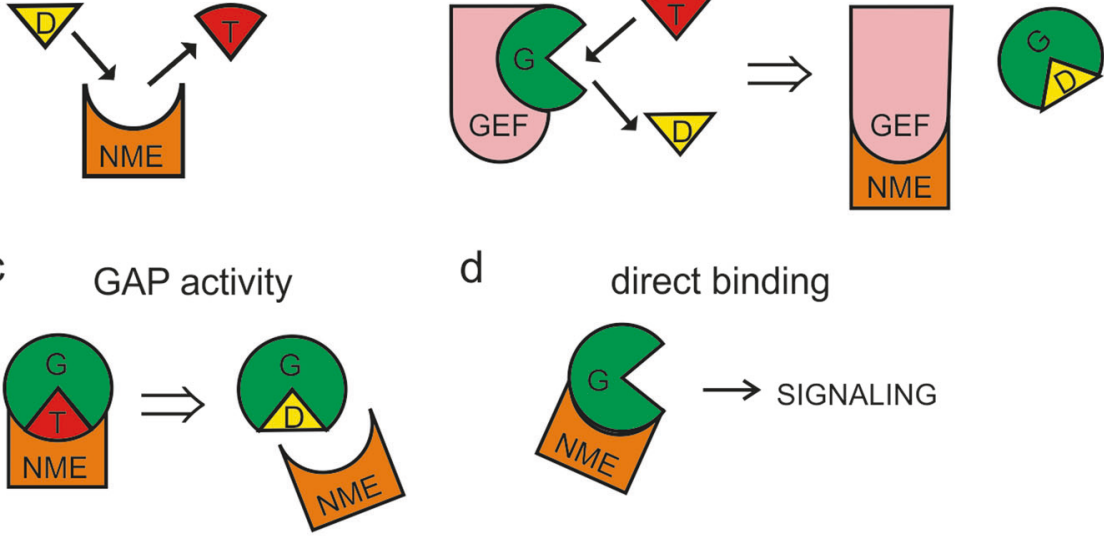

e

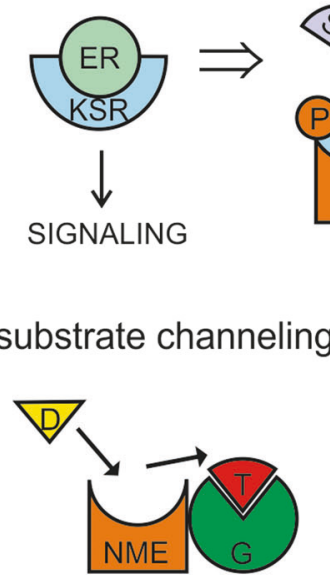

d

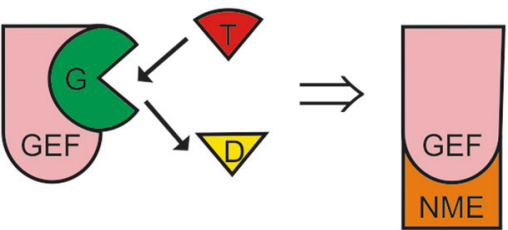

b

\section{GEF inhibition}

direct binding

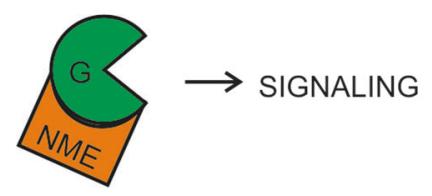
downstream signaling inhibition
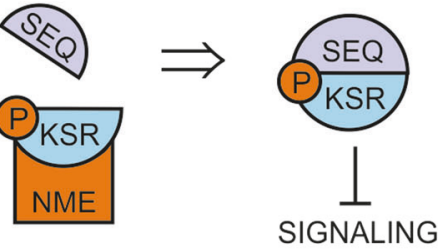

g

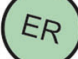

primarily through engaging in protein-protein interactions [17]. Rather than by fine-tuning the local GTP concentration, the GTPase activity is regulated more directly by GEFs and GAPs (GTPase-activating proteins). NMEs interact with specific upstream regulators, such as GEFs, and downstream regulators of Ras GTPases, and in this way modulate the GTPase signaling. We provide a concise overview of these interactions, and interactions of NME proteins with small GTPases themselves. Interestingly, most of these interactions ultimately lead to inhibition of the signal transduction pathways that involve Ras GTPase activity (Table 1).

\section{Inhibition of Rho GEF activity by NME proteins}

Small GTPases from the Rho family regulate a broad range of biological processes, including remodeling of the actin cytoskeleton, cell polarity and motility, endocytosis and vesicle trafficking, cell cycle progression, cell division, and tumorigenesis [18]. The best characterized representatives of the Rho family GTPases are RhoA, Rac1, and Cdc42. Rho family GTPases typically take part in signal transduction cascades by participating in multimolecular complexes that include their regulators, e.g., GEFs, and their effectors [19]. Prominent examples include: Cdc42, its GEF PIX $\alpha$ and its effector p21-activated kinase PAK1 [20], Rac1, its GEF ArhGEF7, cytoskeletal protein coronin 1A, PAK1, and RhoGDI $\alpha$ [21], and RhoA, its GEFs Net1 and p115RhoGEF, scaffold protein CNK1 and components of the JNK MAP kinase cascade [22]. Rac1-specific GEF Tiam1 is known to participate in several complexes regulated by Rac, e.g., with WAVE and IRSp53 [23] and the Par polarity complex [24]. Tiam1 is also localized in large multiprotein complexes that integrate Rac activation with regulatory pathways mediated by other small GTPases and plays a key role in the regulation of Rac activity at epithelial junctions [25-27].

NME proteins modulate the activity of Rho GTPases predominantly through binding to their GEFs, such as Tiam1, Dbl-1, and Lbc (Table 1, GEF inhibition). 


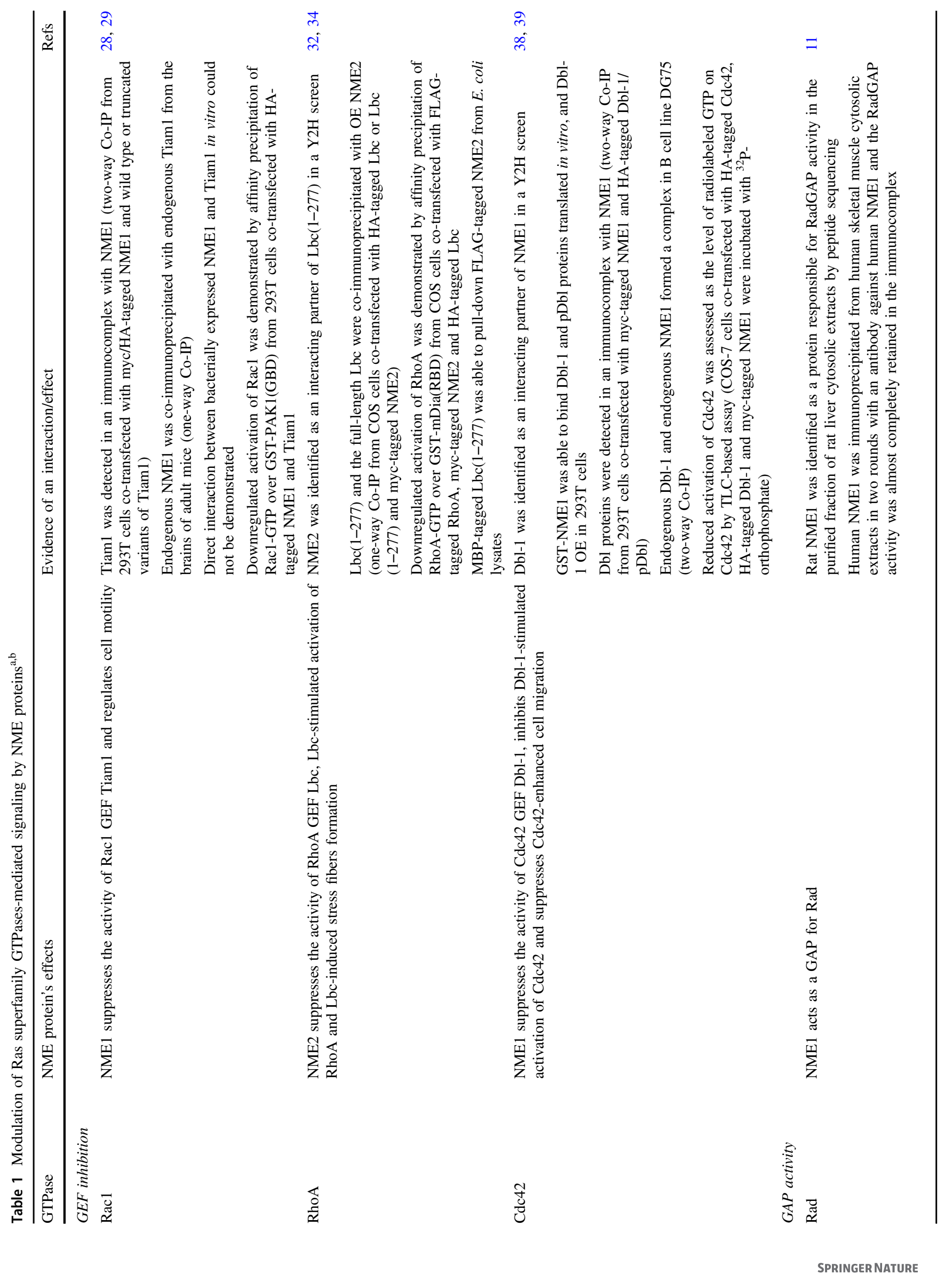




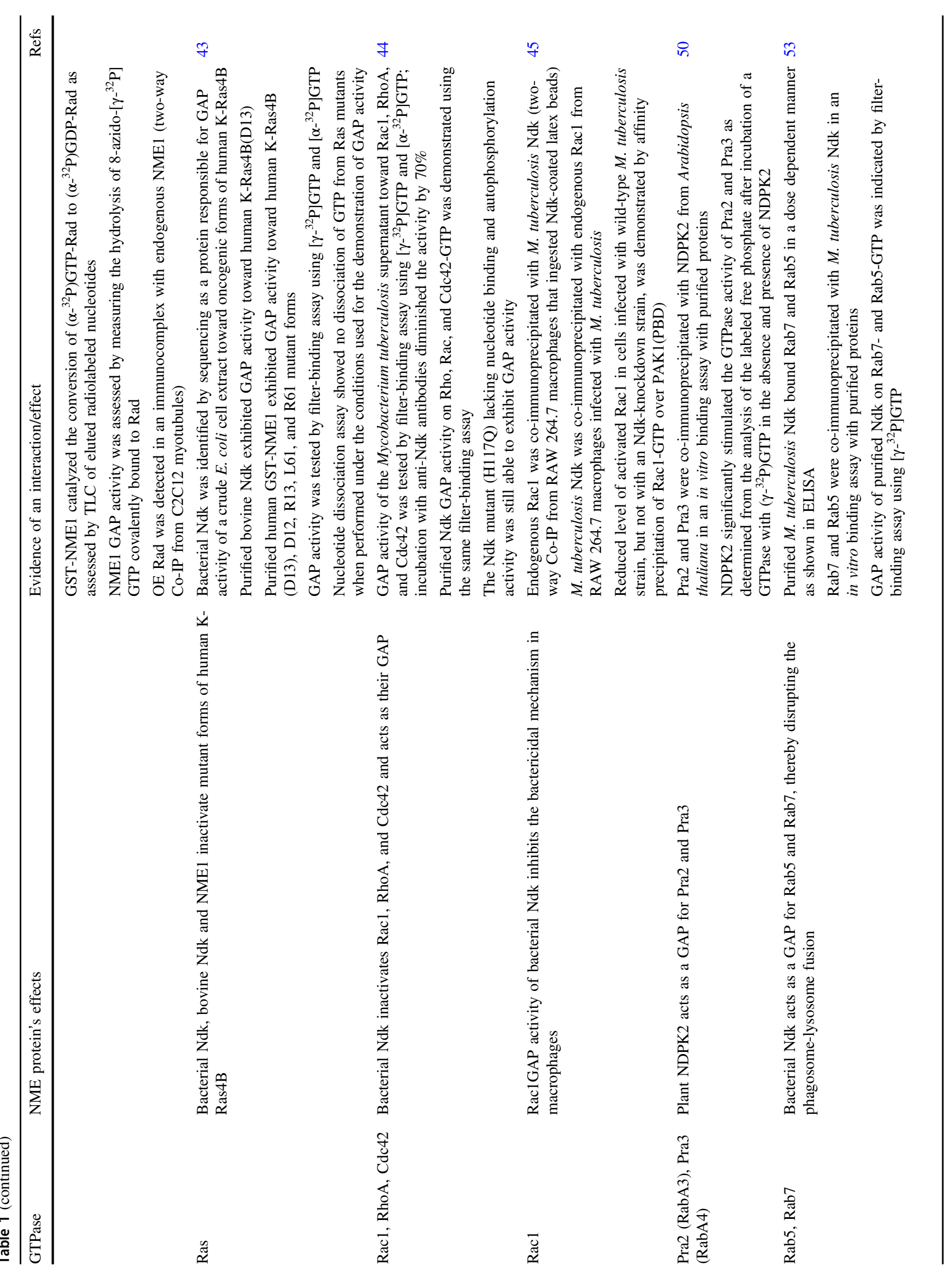




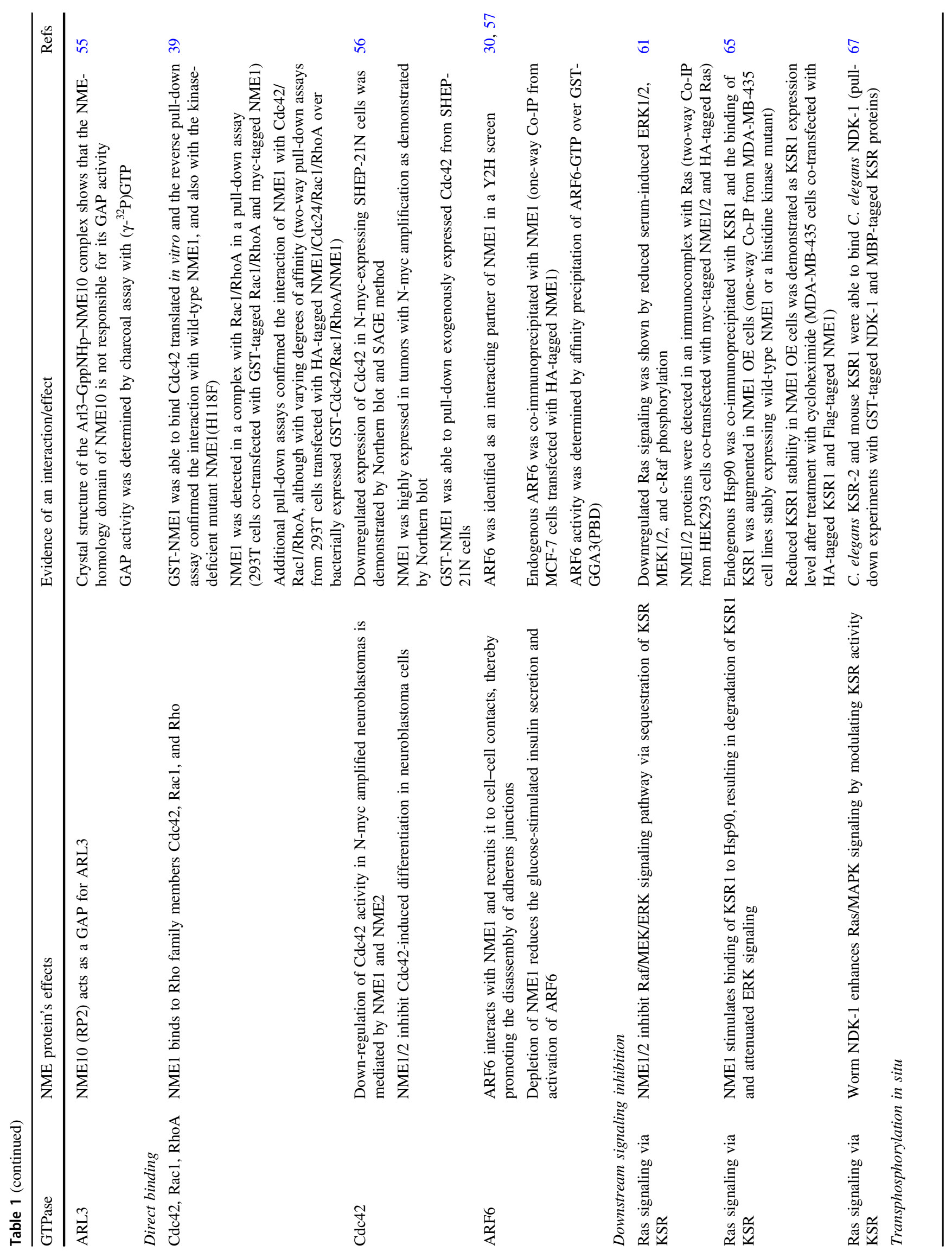




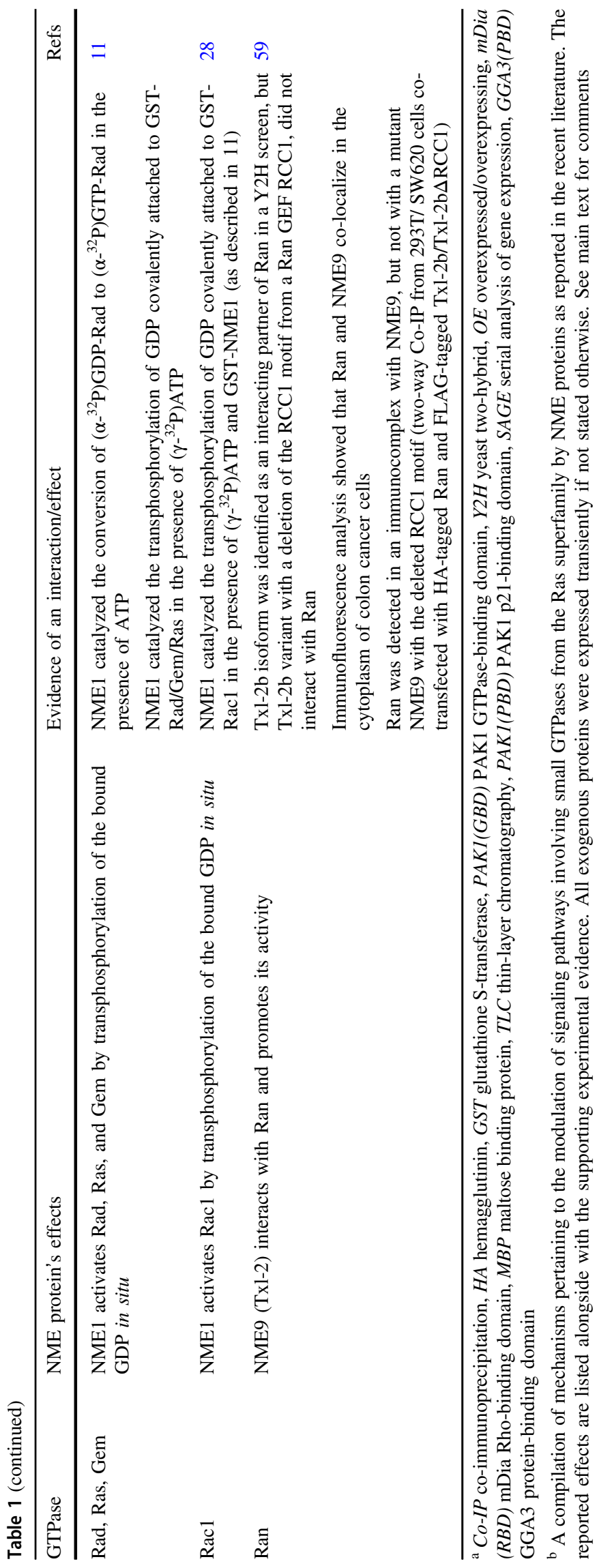

Interestingly, the modulation mechanism appears to be independent of the NDP kinase activity of NME proteins, and instead depends on direct protein-protein interactions between GEFs and NMEs (Fig. 1b). Two-way co-immunoprecipitation experiments using transfected HEK293T cells and mouse brain extracts showed association between NME1 and Tiam1, a GEF specific for Rac1 [28]. The amino-terminal part of Tiam1, Tiam1(N392), was identified as the NME1-binding region and this interaction was independent of the NDP kinase activity of NME1. On the other hand, NME1 mutants lacking the Kpn loop, which is essential for NME oligomerization and modulates its histidine protein kinase activity, could not bind to Tiam1(N392), indicating that either of these activities is necessary for the interaction to occur in vivo. Overexpression of NME1 leads to a decreased level of activated Rac1, and this effect was demonstrated to be a consequence of the interaction between NME1 and Tiam1. Consistent with this negative regulation of Rac1 activity by NME1 is the phenotype of Rat1 fibroblasts expressing the GFP-NME1 construct, which showed reduced formation of membrane ruffles [28]. The suppression of Rac1 activity via binding of NME1 to Tiam1 and subsequent inhibition of its GEF activity was shown to be important for the regulation of cell migration in a study of glioblastoma invasiveness [29]. Also, it was hypothesized that the reduced level of GTP-bound Rac1 in MDCK cells expressing constitutively active form of ARF6 is a consequence of the ARF6-mediated recruitment of NME1 to adherens junctions where it deactivates Tiam1 [30].

Small Rho GTPases are vastly outnumbered by their corresponding GEFs, which are thought to specify the location and timing of Rho activation in response to distinct upstream signals [5]. Multidomain protein Lbc, initially discovered as a proto-oncogene product from human lymphoma, was subsequently characterized as a RhoGEF [31]. A yeast two-hybrid screen and co-immunoprecipitation from COS cells identified NME2 as a specific and NDP kinase-independent binding partner of Lbc [32]. Considering the biological role of Lbc as an activator of RhoA, these authors examined the influence of NME2 on RhoA activation and showed that Lbc stimulates activation of RhoA, whereas this activation was diminished when NME2 was present. Also, expression of Lbc in NIH3T3 fibroblasts induced formation of stress fibers, while NME2 suppressed the Lbc-induced stress fibers formation. These data collectively indicate that NME2 is a negative regulator of the Lbc-stimulated RhoA signaling [32]. In an extension of this study, co-immunoprecipitation from COS cells suggested that NME2, Lbc, and ICAP1- $\alpha$, a protein involved in integrin signaling and a putative guanine dissociation inhibitor (GDI) for Rho GTPases [33], assemble in a tertiary complex in vivo [34]. However, only binary interactions 
between Lbc and ICAP1- $\alpha$ with the FLAG-tagged NME2 could be confirmed using the purified proteins.

Small Rho GTPase Cdc42 regulates cytoskeletal organization and membrane trafficking in cell proliferation, motility, and polarity [35]. Again, multiple Cdc42-GEFs constitute building blocks of multiprotein complexes that confer specificity of Cdc42-mediated signaling in response to external stimuli [36]. Originally isolated from diffuse Bcell lymphoma, Dbl-1 is a prototypical member of the Dbl family of GEF proteins characterized by the presence of tandem Dbl homology (DH) and pleckstrin homology (PH) domains [37]. Dbl-1 was identified as an NME1-binding protein in a yeast two-hybrid screen [38], and this interaction was confirmed by two-way co-immunoprecipitation of endogenously expressed proteins from B lymphoma cell lines and by pull-down assays in which PH domain of Dbl-1 was identified as the region necessary for the interaction $[38,39]$. Since it was known that Dbl-1 acts as a GEF for human $\mathrm{Cdc} 42$, authors explored the influence of NME1 on Cdc42 activation. It was shown that the presence of NME1 reduces GTP loading of $\mathrm{Cdc} 42$ and this effect was not affected when kinase-deficient mutants of NME1 were tested. This result suggests that physical interaction, but not NDP and histidine kinase activity, is important for the suppression of Dbl-1 GEF activity. Another way in which NME1 might suppress the GEF activity of Dbl-1 is by abrogating its phosphorylation, since this modification of Dbl-1 is lost in the presence of NME1 [38].

Functional tests showed that NME1 and Dbl-1 co-localize at the cell edge in HEK293T and COS-7 cells and that overexpression of Dbl-1 induces formation of membrane ruffles [38, 39]. The ruffling was reduced when NME1 was co-expressed with Dbl-1, indicating that NME1 downregulates Dbl-1, which in turn inhibits Dbl-1-stimulated Cdc42 activation in cellulo. Along similar lines, when expressed in breast cancer cell line MDA-MB435, NME1 reduced the ability of these cells to migrate, probably by engaging in an interaction with Dbl-1 and possibly other GEFs [39]. This phenotype could be rescued when Dbl-1 was overexpressed and prevailed over the inhibitory effect of NME1.

\section{GAP activity of NME proteins}

First evidence for the regulation of Ras family GTPases by NMEs was provided for Rad (Ras-associated with diabetes) [11]. Rad, together with REM, REM2, and GEM/Kir, belongs to the REM ( $\mathrm{Rad}$ and Gem-related) or RGK ( $\mathrm{Rad} /$ Gem/Kir) subfamiliy of Ras GTPases [40]. They are characterized by substitutions of conserved amino acid residues inside the G-box and by the absence of prenylation motifs in their carboxy terminus [41]. Regardless, RGK proteins exhibit GTPase activity, but instead undergoing typical lipid modification, their C-terminal extension is responsible for the plasma membrane localization. Biological functions of RGK proteins encompass actin cytoskeletal remodeling via inhibition of Rho signaling, and inhibition of voltagedependent $\mathrm{Ca}^{2+}$ channels by sequestering $\mathrm{CaV} \beta$ subunit and thus interfering with $\mathrm{CaV} \beta-\mathrm{CaV} \alpha 1$ association [42].

NME1 was identified in a search for a protein that is responsible for a putative RadGAP activity in cytosolic extracts of rat liver cells (Fig. 1c) [11]. Using coimmunoprecipitation assay it was shown that NME1 interacts with $\operatorname{Rad}$ in vivo (Table 1, GAP activity). After demonstrating an increased conversion of radiolabeled GTP to GDP bound to Rad in the presence of NME1, the authors tested whether GTP hydrolysis occurs in situ. In order to prevent its dissociation from Rad, 8-azido- $\left[\gamma^{32} \mathrm{P}\right] \mathrm{GTP}$ was covalently linked to Rad by a short exposure to UV light and addition of NME1 led to a reduction of radiolabeled Rad-bound GTP. Decrease of radioactivity bound to $\mathrm{Rad}$ upon addition of NME1 was taken as a measure of its RadGAP activity. However, a prolonged exposure to UV light resulted in increased binding of radiolabeled GTP to Rad, but to a decreasing GTP hydrolysis upon addition of NME1. This effect was interpreted as a consequence of the UV light-induced protein denaturation. However, it was suggested that short exposures to UV light did not result in sufficient cross-linking of radiolabeled GTP to Rad [14]. Therefore, the detected GTP hydrolysis might have occurred by the NDP kinase activity of NME1 in solution.

Bacterial Ndk was identified as a protein responsible for the RasGAP activity in crude $E$. coli extracts, capable of inactivating several oncogenic forms of Ras that are most commonly detected in human cancers [43]. The same activity was then demonstrated for its mammalian orthologues, bovine Ndk and human NME1, which were also able to promote GTP hydrolysis on mutant forms of Ras. At the same time, Ndk could not increase GTPase activity of wildtype Ras, which is in agreement with previous observations that NME is not a RasGAP [11]. These results suggested that loss of NME proteins could increase tumorigenicity of Ras-driven tumors.

Another prokaryotic NDP kinase, Ndk from Mycobacterium tuberculosis, was identified as a protein that stimulates intrinsic GTP-hydrolysis activity of Rac1, RhoA, and Cdc42 in vitro, thus acting as a GAP for Rho GTPases [44]. Efficiency of GTP to GDP conversion was almost identical for all three GTPases. In a follow-up study, to further characterize the interaction between Ndk and Rho GTPases, RAW 264.7 macrophages were infected with bacteria or allowed to ingest Ndk-coated latex beads [45]. Analysis of macrophage lysates by two-way co-immunoprecipitation revealed an association of bacterial Ndk with Rac1, but not with RhoA and Cdc42. Rac1GAP activity of $\mathrm{Ndk}$ in macrophages infected with wild-type bacteria was 
indicated by a dramatically reduced level of Rac1-GTP, while this effect was not detectable in macrophages infected with M. tuberculosis strain with reduced Ndk expression. In terms of bacterial virulence mechanism, Ndk inhibits formation of phagocyte NADPH oxidase (NOX2) complex responsible for the production of ROS [46]. It was proposed that this inhibition is achieved via Ndk-mediated inactivation of Rac1, since Rac1 is one of the subunits of NOX2 complex and its activation is necessary for membrane recruitment of another NOX2 subunit, p67phox [45].

Rab GTPases are known to regulate the intracellular transport of membranes and vesicle trafficking in plants and other organisms [47, 48]. Similar to NME proteins in mammals, NDPK isoforms in plants also perform diverse important roles [49]. NDPK2 from Arabidopsis thaliana was proposed to function as a GAP for small GTPases Pra2 (RabA3) and Pra3 (RabA4), which belong to the Rab family [50]. Active Rab5 and Rab7 play crucial roles in promoting the interaction of vesicles with lysosomes [51, 52]. It was shown that Ndk from M. tuberculosis induces GTPhydrolysis on active Rab5 and Rab7 in a cell-free biochemical assay [53]. It was proposed in this work that the GAP activity of Ndk, released from pathogenic mycobacteria within the phagosome, blocks the fusion of the phagosome with lysosomes and thereby protects bacteria from degradation. Interestingly, the $D$. melanogaster NME1/2 homolog Awd was shown to be required for Rab5 function in promoting maturation of early endosomes [54].

Despite a number of reports that provide evidence for an NME GAP activity, in our opinion this activity still awaits the definitive confirmation. The filter-binding assays suffer from potential technical flows described in the case of Rad, whereas the functional consequences of NME-induced GEF inhibition might phenocopy the GAP activation. It is instructive to mention here an example where a member of the group II NMEs, which lack the NDP kinase activity, XRP2 (NME10), was identified as a GAP for the small G protein ARL3 (Arf-like 3) [55]. Crystal structure of the Arl3-GppNHp-NME10 complex was solved showing that the ARL3/NME10 interaction surface, which is responsible for the GAP activity of NME10, resides in the tubulinspecific chaperone C-homology domain of NME10 and not in its NME-homology domain, shared by all NME proteins. A similar mechanistic approach would be invaluable to vindicate the alleged untypically non-selective GAP activity of group I NMEs toward diverse small GTPases (Table 1, GAP activity).

\section{Direct interactions between NME proteins and small GTPases}

A direct interaction between NME1 and the three prototypical members of the Rho family, Cdc42, Rho, and Rac1, was demonstrated using two-way pull-down assays with tagged proteins expressed in $293 \mathrm{~T}$ cells (Fig. 1d; Table 1, direct binding) [39]. An interaction between NME proteins and $\mathrm{Cdc} 42$ was also suggested in neuroblastoma [56]. These authors detected by a pull-down experiment that NME1 and Cdc42 can interact in SHEP-21N neuroblastoma cells. Nmyc amplification in these cells downregulates expression and activity of $\mathrm{Cdc} 42$ and inhibits Cdc42-induced differentiation. In cells with reduced $\mathrm{N}$-myc expression, Cdc42induced differentiation was re-established, but introduction of NME1 or NME2 again inhibited differentiation, indicating that the regulation of $\mathrm{Cdc} 42$ activity by $\mathrm{N}$-myc is mediated by NME proteins. In another study, however, no direct association of NME1 with Rho family GTPases was detected in vitro [28]. Such conflicting results indicate the extreme caution needed to differentiate between the direct interaction of small GTPases with NME proteins and a binding mediated by a corresponding GEF-containing complex, as illustrated also in the next example.

ARF6, a member of the ARF family of small GTPases, interacts with NME1 and recruits it to cell-cell contacts thus promoting disassembly of adherens junctions in MCDK II cells [30]. Importantly, overexpression of an NDP kinasedead mutant of NME1, but not of the wild-type NME1, inhibited the disassembly of adherens junctions, suggesting a critical role for NME1 NDP kinase activity in this process. Consistent with this finding, it was established that the disassembly is driven by dynamin-dependent vesicle internalization, a process in which NME serves as a supplier of GTP [9]. Therefore, it appears that ARF6 serves as a vehicle that transports NME to the membrane at cell-cell adhesion sites and thereby facilitates dynamin-dependent endocytosis of E-cadherin. Another mechanism by which active ARF6 might further promote disassembly of adherens junctions is downregulation of active Rac1 by NME1-mediated sequestration of Tiam1 [28]. Indeed, the level of GTPbound Rac1 is reduced in MDCK cells expressing constitutively active form of ARF6, indicating that ARF6mediated recruitment of NME1 to the membrane is important for the regulation of Rac1 activity [30]. It was also suggested that NME1 can act upstream of ARF6 in pancreatic beta cells, where knockdown of NME1 diminished the glucose-stimulated activation of ARF6 [57].

It was shown that expression of an NME protein from the group II, Txl-2 (NME9), is increased in colon cancer [58]. The following analyses indicated that its most abundant isoform, Txl-2b, interacts with the small GTPase Ran and promotes its activity in tumor cell invasion [59]. This result is consistent with the previous finding that Ran is a downstream effector of the PI3K signaling pathway involved in invasion and metastasis of cancer cells [60]. Although the interaction between Ran and $\mathrm{Tlx}-2 \mathrm{~b}$ was dependent on a motif from RanGEF RCC1, no direct 
demonstration of a putative Txl-2b GEF activity was provided.

\section{Inhibition of signaling downstream of Ras}

Besides regulating Ras family GTPases by means of a direct interaction, NME proteins are involved in the modulation of Ras-mediated signaling pathways via binding to downstream regulators of Ras activity (Fig. 1e; Table 1, downstream signaling inhibition). Overexpression of RGS19, member of the regulators of G-protein signaling family (RGS proteins), in HEK293 cells suppresses Ras signaling via upregulation of NME1/2 and subsequent augmented phosphorylation of the kinase suppressor of Ras (KSR) at Ser392 [61]. KSR acts as a scaffold for Ras/Raf/MEK/ERK signaling pathway and is required for efficient signal propagation [62]. NME1 is one of the kinases responsible for the phosphorylation of KSR at Ser392, and this modification confers binding to 14-3-3 and cytoplasmic sequestration of KSR that efficiently precludes formation of the Ras signaling complex at the plasma membrane [63, 64]. In addition, it was demonstrated that NME1 overexpression in breast carcinoma cells stimulates binding of KSR to the chaperone protein Hsp90, which results in enhanced degradation of KSR and, consequently, attenuated ERK signaling [65]. Consistently, overexpression of NME2 inhibited the Rasmediated activation of the ERK pathway in NIH3T3 and HEK293 cells, probably by an analogous mechanism [66]. Interestingly, a related mechanism of NME-mediated modulation of Ras signaling was identified in C. elegans [67]. A single-group I NME homolog in this organism, NDK-1, also binds to KSR, but in contrast to results obtained in mammalian cell lines, this interaction enhances the Ras/MAPK signaling.

\section{Substrate chanelling and transphosphorylation in situ hypotheses}

A hypothesis was formulated early on that proposed a focused delivery of NME-generated NTPs directly to their site of action (Fig. 1f) [68]. Such process would resemble the substrate channeling mechanism in which an intermediate product is transferred between closely apposed active sites of enzymes that catalyze sequential reactions in several biosynthetic pathways [69]. It was recently shown that members of the group I NMEs interact directly with dynamin superfamily proteins and supply them with GTP, thereby supporting their function in membrane remodeling [9]. Dynamin GTPases are molecular motors that utilize hydrolysis of GTP as a fuel, and as such have a high intrinsic GTP-hydrolysis rate [70]. Together with their relatively low-binding affinity for GTP, dynamins' activity is poised to depend on a high local GTP concentration provided by NMEs.

In contrast to dynamins, Ras superfamily GTPases have low intrinsic GTP-hydrolysis rate and high-binding affinity for both GTP and GDP [40]. Further, Ras GTPases are signaling molecules and not molecular motors, and their activity is exerted in their GTP-bound state; GTP is used here as a co-factor and not as an energy fuel. It is therefore not surprising that NME proteins do not appear to regulate Ras GTPases by a direct, high-rate supply of GTP [14]. As an alternative mechanism of NDP kinase-mediated regulation of small GTPases, direct phosphorylation of GDP bound to inactive GTPase was proposed (Fig. 1g) [11, 28]. However, due to constitutive NDP transphosphorylation activity of NME proteins it is difficult to experimentally differentiate whether the GDP- transphosphorylation occurs in situ or takes place in solution after dissociation of GDP from the GTPase. For example, it was reported that NME1 can catalyze reloading of Rad, Gem, and Ras in situ by transphosphorylation of the bound GDP, thus mimicking function of a GEF (Table 1, transphosphorylationin situ) [11]. However, this mechanism was disputed since both the phosphohistidine that serves as a phosphate donor of NDP kinases and the Ras-bound GDP are buried deep inside shielded regions of the respective proteins, making a direct interaction between the phosphate donor and acceptor unlikely [14, 15]. Also, the method used to prevent dissociation of GDP from Rad during the transphosphorylation assay was criticized to be prone to artifacts. Namely, the crosslinking of GDP to small GTPases by a prolonged exposure to UV light might have led to protein denaturation. Since group I NME proteins are able to phosphorylate denatured proteins [71], it was suggested that the transfer of radioactive phosphate was not specific to GDP [14].

\section{Concluding remarks}

Members of the NME family interact with numerous proteins that regulate Ras superfamily GTPases or mediate their signaling $[17,72]$. Since NDP kinases are involved in the production of GTP, the major cofactor of Ras GTPases, it was hypothesized that group I NMEs would stimulate activity of small GTPases [73]. Contrary to these early expectations, it turned out that the majority of interactions inhibit Ras-mediated signal transduction pathways (Table 1). There are also examples in the literature where a single-protein pair was reported to engage in interactions that have opposite effects on the activity of the involved GTPase (NME1 and Rad) [11], or where similar interactions result in opposite outcomes depending on the model organism (KSR in mammals vs. worms) [61, 67].

A number of interdependencies between NME proteins and Rho GTPase activities was reported without providing 
evidence for underlying mechanisms. It was shown that glucose-induced association of active Rac1 with the membrane in pancreatic beta cells depends on NME1 kinase activity, but not on its exonuclease activity [57]. In an example of apparently negative regulation of Rac1 by NMEs, silencing of NME1 in hepatoma cell line HepG2 increased mean and maximal cell velocity, elevated the level of GTP-bound Rac1, and enhanced formation of filopodia [74]. Also, NME2 was implicated in formation of a complex with thromboxane A2 receptor $\beta$ (TP $\beta)$ involved in the Rac1-mediated endocytosis of a $G$ protein-coupled receptor [75]. It was shown that Rad enhances cell growth in a breast cancer cell line, and that this growth-promoting effect was abolished by the presence of NME1 [76]. The same effect was demonstrated in vivo; mice injected with cells overexpressing Rad developed larger tumors than the control group and the tumor growth-promoting effect was absent when injected cells co-expressed NME1. In Dictyostelium discoideum, NDPK-C was shown to localize to vesicles in prespore cells during multicellular development of this organism [77]. Since the GTPase Rab7 was also enriched in these vesicles, it was hypothesized that NDP kinase could be providing GTP for Rab7. Rab7 was also shown to activate the fusion of early endosomes in a reconstituted system in vitro, and NDP kinase in this case provided the GTP necessary for Rab7 activity [78].

In addition to its engagement in protein-protein interactions and kinase activities, NME1 also displays the $3^{\prime}-5^{\prime}$ exonuclease activity known to play an important role during DNA replication and repair [79]. Potential influence of this activity on the mRNA expression patterns was tested by microarray analysis in melanoma cell lines with augmented or reduced levels of NME1 expression [80]. The results point to a transcriptional regulation by NME1 of three proteins that modulate signaling pathways mediated by Ras superfamily GTPases: Lipocalin-2, BRAP (BRCA1associated protein) and IQGAP2. Interestingly, an inhibitory effect of the NME1 3'-5' exonuclease activity on the involved Ras and Rho-mediated signaling pathways was indicated for all three proteins.

Although, NMEs are predominantly cytosolic proteins, their interaction with small GTPases is expected to occur at the membrane [81, 82]. Two recent studies analyzed properties related to membrane binding of NME1, NME2, and NME4 isoenzymes [83, 84]. NME1 was not able to bind directly to model membranes, indicating that a partner, possibly a small GTPase, is required for its association with membranes in vivo. On the other hand, NME2 and NME4 both bound efficiently to liposomes, and whereas NME2 binding necessarily involves an anionic phospholipid partner, NME4 can bind either zwitterionic or anionic phospholipids [83]. It was further shown that the membrane binding of NME2 occurs in two steps leading to a local decrease in membrane fluidity and formation of protein patches [84]. It was suggested that this propensity of NME2 to form microdomains might be relevant for specific protein-protein interactions occurring at the plasma cell membrane.

Besides the regulation of Ras GTPases by NME proteins, there are examples where small GTPases are influencing the function of NMEs. As already referred to, ARF6 facilitates recruitment of NME1 to the cell-cell adhesion sites [30], and might play the role of a non-lipid partner needed for binding of NME1 to the membrane [83]. Also, it was proposed that Rad can enhance the NDP kinase activity of NME1 and reduce its autophosphorylation on histidine residues [11]. Another example is presented in a study of migration and apoptotic engulfment of distal tip cells in C. elegans, where it was shown that the single-worm group I NME homolog NDK1 is required for both processes and acts downstream of Rac1 and RhoG in this organism [85].

In conclusion, it is apparent that NME proteins modulate the activity of small GTPases from the Ras superfamily and related signaling pathways in diverse ways. However, many of the proposed mechanisms are still not substantiated or fully resolved. In order to clarify the open questions in the field, a multifaceted approach, including both biochemical and physiological assays is recommended. Specifically, protein interaction studies should be performed using endogenous proteins, and aim at defining the interaction domains. Further, underrepresented fluorescent techniques used to identifying protein-protein interactions in live cells, such as assays based on fluorescence resonance energy transfer and bimolecular fluorescence complementation, should be used to complement protein biochemistry. Fluorescence-based approaches could also provide additional information about the subcellular compartments where the investigated proteins exert their biological functions. These studies should be accompanied by comprehensive characterization of functional interactions between NMEs and small GTPases in model organisms. It should be appreciated, however, that NME's pleiotropic effects and functional redundancies between numerous isoforms, especially in mammals, are hampering mechanistic studies. Therefore, parallel studies in non-mammalian model organisms have a prospect to provide a better insight into interrelatedness between these two groups of proteins.

Acknowledgements This work was supported by the Croatian Science Foundation under the project IP-2014-09-4753. We thank Dr. Maja Herak Bosnar for critical reading of the manuscript.

\section{Compliance with Ethical Standards}

Conflict of interest The authors declare that they have no conflict of interest. 


\section{References}

1. Goitre L, Trapani E, Trabalzini L, et al. The ras superfamily of small GTPases: the unlocked secrets. Methods Mol Biol. 2014;1120:1-18.

2. Wennerberg K, Rossman KL, Der CJ. The Ras superfamily at a glance. J Cell Sci. 2005;118:843-6.

3. Cherfils J, Chardin P. GEFs: structural basis for their activation of small GTP-binding proteins. Trends Biochem Sci. 1999;24:306-11.

4. Hennig A, Markwart R, Esparza-Franco MA, et al. Ras activation revisited: role of GEF and GAP systems. Biol Chem. 2015;396:831-48.

5. Rossman KL, Der CJ, Sondek J. GEF means go: turning on RHO GTPases with guanine nucleotide-exchange factors. Nat Rev Mol Cell Biol. 2005;6:167-80.

6. Bos JL, Rehmann H, Wittinghofer A. GEFs and GAPs: critical elements in the control of small $G$ proteins. Cell. 2007;129:865-77.

7. Lacombe ML, Milon L, Munier A, et al. The human Nm23/ nucleoside diphosphate kinases. $\mathrm{J}$ Bioenerg Biomembr. 2000;32:247-58.

8. Agarwal RP, Robison B, Parks RE. Nucleoside diphosphokinase from human erythrocytes. Methods Enzymol. 1978;51:376-86.

9. Boissan M, Montagnac G, Shen Q, et al. Membrane trafficking. Nucleoside diphosphate kinases fuel dynamin superfamily proteins with GTP for membrane remodeling. Science. 2014;344:1510-5.

10. Randazzo PA, Northup JK, Kahn RA. Activation of a small GTPbinding protein by nucleoside diphosphate kinase. Science. 1991;254:850-3.

11. Zhu J, Tseng YH, Kantor JD, et al. Interaction of the Ras-related protein associated with diabetes $\mathrm{rad}$ and the putative tumor metastasis suppressor NM23 provides a novel mechanism of GTPase regulation. Proc Natl Acad Sci USA. 1999;96:14911-8.

12. Randazzo PA, Kahn RA, Northup JK. Nucleoside diphosphate kinase: conclusions withdrawn. Science. 1992;257:862.

13. Randazzo PA, Northup JK, Kahn RA. Regulatory GTP-binding proteins (ADP-ribosylation factor, Gt, and RAS) are not activated directly by nucleoside diphosphate kinase. J Biol Chem. 1992;267:18182-9.

14. Otero AS. NM23/nucleoside diphosphate kinase and signal transduction. J Bioenerg Biomembr. 2000;32:269-75.

15. Hippe H-J, Wieland T. High energy phosphate transfer by NDPK B/Gbetagammacomplexes-an alternative signaling pathway involved in the regulation of basal cAMP production. J Bioenerg Biomembr. 2006;38:197-203.

16. Steeg PS, Zollo M, Wieland T. A critical evaluation of biochemical activities reported for the nucleoside diphosphate kinase/ Nm23/Awd family proteins: opportunities and missteps in understanding their biological functions. Naunyn Schmiede Arch Pharmacol. 2011;384:331-9.

17. Marino N, Marshall J-C, Steeg PS. Protein-protein interactions: a mechanism regulating the anti-metastatic properties of Nm23-H1. Naunyn Schmiede Arch Pharmacol. 2011;384:351-62.

18. Hodge RG, Ridley AJ. Regulating Rho GTPases and their regulators. Nat Rev Mol Cell Biol. 2016;17:496-510.

19. Buchsbaum RJ. Rho activation at a glance. J Cell Sci. 2007;120:1149-52.

20. Li Z, Hannigan M, Mo Z, et al. Directional sensing requires G beta gamma-mediated PAK1 and PIX alpha-dependent activation of Cdc42. Cell. 2003;114:215-27.

21. Castro-Castro A, Ojeda V, Barreira M, et al. Coronin 1A promotes a cytoskeletal-based feedback loop that facilitates Rac1 translocation and activation. EMBO J. 2011;30:3913-27.
22. Jaffe AB, Hall A, Schmidt A. Association of CNK1 with Rho guanine nucleotide exchange factors controls signaling specificity downstream of Rho. Curr Biol. 2005;15:405-12.

23. Connolly BA, Rice J, Feig LA, et al. Tiam1-IRSp53 complex formation directs specificity of rac-mediated actin cytoskeleton regulation. Mol Cell Biol. 2005;25:4602-14.

24. Pegtel DM, Ellenbroek SIJ, Mertens AEE, et al. The Par-Tiam1 complex controls persistent migration by stabilizing microtubuledependent front-rear polarity. Curr Biol. 2007;17:1623-34.

25. Guillemot L, Paschoud S, Jond L, et al. Paracingulin regulates the activity of Rac1 and RhoA GTPases by recruiting Tiam1 and GEF-H1 to epithelial junctions. Mol Biol Cell. 2008;19:4442-53.

26. Goicoechea SM, Awadia S, Garcia-Mata R. I'm coming to GEF you: regulation of RhoGEFs during cell migration. Cell Adhes Migr. 2014;8:535-49.

27. Citi S, Guerrera D, Spadaro D, et al. Epithelial junctions and Rho family GTPases: the zonular signalosome. Small GTPases. 2014;5:1-15.

28. Otsuki Y, Tanaka M, Yoshii S, et al. Tumor metastasis suppressor nm23H1 regulates Rac1 GTPase by interaction with Tiam1. Proc Natl Acad Sci USA. 2001;98:4385-90.

29. Tanaka M, Kuriyama S, Aiba N. Nm23-H1 regulates contact inhibition of locomotion, which is affected by ephrin-B1. J Cell Sci. 2012;125:4343-53.

30. Palacios F, Schweitzer JK, Boshans RL, et al. ARF6-GTP recruits Nm23-H1 to facilitate dynamin-mediated endocytosis during adherens junctions disassembly. Nat Cell Biol. 2002;4:929-36.

31. Sterpetti P, Hack AA, Bashar MP, et al. Activation of the Lbc Rho exchange factor proto-oncogene by truncation of an extended $\mathrm{C}$ terminus that regulates transformation and targeting. Mol Cell Biol. 1999;19:1334-45.

32. Iwashita S, Fujii M, Mukai H, et al. Lbc proto-oncogene product binds to and could be negatively regulated by metastasis suppressor nm23-H2. Biochem Biophys Res Commun. 2004;320:1063-8.

33. Degani S, Balzac F, Brancaccio M, et al. The integrin cytoplasmic domain-associated protein ICAP-1 binds and regulates Rho family GTPases during cell spreading. J Cell Biol. 2002;156:377-87.

34. Miyamoto M, Iwashita S, Yamaguchi S, et al. Role of $n m 23$ in the regulation of cell shape and migration via Rho family GTPase signals. Mol Cell Biochem. 2009;329:175-9.

35. Melendez J, Grogg M, Zheng Y. Signaling role of Cdc42 in regulating mammalian physiology. J Biol Chem. 2011;286: 2375-81.

36. Sinha S, Yang W. Cellular signaling for activation of Rho GTPase Cdc42. Cell Signal. 2008;20:1927-34.

37. Cook DR, Rossman KL, Der CJ. Rho guanine nucleotide exchange factors: regulators of Rho GTPase activity in development and disease. Oncogene. 2014;33:4021-35.

38. Murakami M, Meneses PI, Knight JS, et al. Nm23-H1 modulates the activity of the guanine exchange factor Dbl-1. Int $\mathrm{J}$ Cancer. 2008;123:500-10.

39. Murakami M, Meneses PI, Lan K, et al. The suppressor of metastasis Nm23-H1 interacts with the Cdc42 Rho family member and the pleckstrin homology domain of oncoprotein Dbl-1 to suppress cell migration. Cancer Biol Ther. 2008;7:677-88.

40. Colicelli J. Human RAS superfamily proteins and related GTPases. Sci STKE 2004;2004:RE13.

41. Neely A, Hidalgo P. Structure-function of proteins interacting with the $\alpha 1$ pore-forming subunit of high-voltage-activated calcium channels. Front Physiol. 2014;5:209.

42. Correll RN, Pang C, Niedowicz DM, et al. The RGK family of GTP-binding proteins: regulators of voltage-dependent calcium channels and cytoskeleton remodeling. Cell Signal. 2008;20:292-300. 
43. Fischbach MA, Settleman J. Specific biochemical inactivation of oncogenic Ras proteins by nucleoside diphosphate kinase. Cancer Res. 2003;63:4089-94.

44. Chopra P, Koduri H, Singh R, et al. Nucleoside diphosphate kinase of Mycobacterium tuberculosis acts as GTPase-activating protein for Rho-GTPases. FEBS Lett. 2004;571:212-6.

45. Sun J, Singh V, Lau A, et al. Mycobacterium tuberculosis nucleoside diphosphate kinase inactivates small GTPases leading to evasion of innate immunity. PLoS Pathog. 2013;9:e1003499.

46. Bedard K, Krause K-H. The NOX family of ROS-generating NADPH oxidases: physiology and pathophysiology. Physiol Rev. 2007;87:245-313.

47. Rehman RU and Sansebastiano G-PD. Plant Rab GTPases in Membrane Trafficking and Signalling. In: Hakeem KR, Rehman RU, Tahir I, editors. Plant signaling: understanding the molecular crosstalk. 1st ed. New Delhi, India, Springer, 2014. p. 51-73.

48. Stenmark H. Rab GTPases as coordinators of vesicle traffic. Nat Rev Mol Cell Biol. 2009;10:513-25.

49. Dorion S, Rivoal J. Clues to the functions of plant NDPK isoforms. Naunyn Schmiede Arch Pharmacol. 2015;388:119-32.

50. Shen Y, Han Y-J, Kim J-I, et al. Arabidopsis nucleoside diphosphate kinase-2 as a plant GTPase activating protein. BMB Rep. 2008;41:645-50.

51. Haas AK, Fuchs E, Kopajtich R, et al. A GTPase-activating protein controls Rab5 function in endocytic trafficking. Nat Cell Biol. 2005;7:887-93.

52. Harrison RE, Bucci C, Vieira OV, et al. Phagosomes fuse with late endosomes and/or lysosomes by extension of membrane protrusions along microtubules: role of Rab7 and RILP. Mol Cell Biol. 2003;23:6494-506.

53. Sun J, Wang X, Lau A, et al. Mycobacterial nucleoside diphosphate kinase blocks phagosome maturation in murine RAW 264.7 macrophages. PLoS ONE. 2010;5:e8769.

54. Ignesti M, Barraco M, Nallamothu G, et al. Notch signaling during development requires the function of awd, the Drosophila homolog of human metastasis suppressor gene Nm23. BMC Biol. 2014;12:12.

55. Veltel S, Gasper R, Eisenacher E, et al. The retinitis pigmentosa 2 gene product is a GTPase-activating protein for Arf-like 3. Nat Struct Mol Biol. 2008;15:373-80.

56. Valentijn LJ, Koppen A, van Asperen R, et al. Inhibition of a new differentiation pathway in neuroblastoma by copy number defects of N-myc, $\mathrm{Cdc} 42$, and nm23 genes. Cancer Res. 2005;65:3136-45.

57. Veluthakal R, Kaetzel D, Kowluru A. Nm23-H1 regulates glucose-stimulated insulin secretion in pancreatic $\beta$-cells via Arf6Rac1 signaling axis. Cell Physiol Biochem Int J Exp Cell Physiol Biochem Pharmacol. 2013;32:533-41.

58. Lu Y, Wang X, Liu Z, et al. Identification and distribution of thioredoxin-like 2 as the antigen for the monoclonal antibody MC3 specific to colorectal cancer. Proteomics. 2008;8:2220-9.

59. Lu Y, Zhao X, Li K, et al. Thioredoxin-like protein 2 is overexpressed in colon cancer and promotes cancer cell metastasis by interaction with ran. Antioxid Redox Signal. 2013;19:899-911.

60. Kurisetty VV, Johnston PG, Johnston N, et al. RAN GTPase is an effector of the invasive/metastatic phenotype induced by osteopontin. Oncogene. 2008;27:7139-49.

61. Tso PH, Wang Y, Yung LY, et al. RGS19 inhibits Ras signaling through Nm23H1/2-mediated phosphorylation of the kinase suppressor of Ras. Cell Signal. 2013;25:1064-74.

62. Nguyen A, Burack WR, Stock JL, et al. Kinase suppressor of Ras (KSR) is a scaffold which facilitates mitogen-activated protein kinase activation in vivo. Mol Cell Biol. 2002;22:3035-45.
63. Hartsough MT, Morrison DK, Salerno M, et al. Nm23-H1 metastasis suppressor phosphorylation of kinase suppressor of Ras via a histidine protein kinase pathway. $J$ Biol Chem. 2002;277:32389-99.

64. Müller J, Ory S, Copeland T, et al. C-TAK1 regulates Ras signaling by phosphorylating the MAPK scaffold, KSR1. Mol Cell. 2001;8:983-93.

65. Salerno M, Palmieri D, Bouadis A, et al. Nm23-H1 metastasis suppressor expression level influences the binding properties, stability, and function of the kinase suppressor of Ras1 (KSR1) Erk scaffold in breast carcinoma cells. Mol Cell Biol. 2005;25:1379-88.

66. Lee M-Y, Jeong W-J, Oh J-W, et al. NM23H2 inhibits EGF- and Ras-induced proliferation of NIH3T3 cells by blocking the ERK pathway. Cancer Lett. 2009;275:221-6.

67. Masoudi N, Fancsalszky L, Pourkarimi E, et al. The NM23-H1/ $\mathrm{H} 2$ homolog NDK-1 is required for full activation of Ras signaling in C. elegans. Development. 2013;140:3486-95.

68. Boissan M, Dabernat S, Peuchant E, et al. The mammalian Nm23/ NDPK family: from metastasis control to cilia movement. Mol Cell Biochem. 2009;329:51-62.

69. Miles EW, Rhee S, Davies DR. The molecular basis of substrate channeling. J Biol Chem. 1999;274:12193-6.

70. Daumke O, Praefcke GJK. Invited review: mechanisms of GTP hydrolysis and conformational transitions in the dynamin superfamily. Biopolymers. 2016;105:580-93.

71. Engel M, Véron M, Theisinger B, et al. A novel serine/threoninespecific protein phosphotransferase activity of Nm23/nucleosidediphosphate kinase. Eur J Biochem. 1995;234:200-7.

72. Vlatković N, Chang S-H, Boyd MT. Janus-faces of NMEoncoprotein interactions. Naunyn Schmiede Arch Pharmacol. 2015;388:175-87.

73. Kimura N. Role of Nucleoside Diphosphate Kinase in G-Protein Action. In: Dickey BF, Birnbaumer L, editors. GTPases in Biology II. 1st ed. Berlin: Springer Berlin Heidelberg; 1993. p. 485-498.

74. Boissan M, De Wever O, Lizarraga F, et al. Implication of metastasis suppressor NM23-H1 in maintaining adherens junctions and limiting the invasive potential of human cancer cells. Cancer Res. 2010;70:7710-22.

75. Rochdi MD, Laroche G, Dupré E, et al. Nm23-H2 interacts with a $G$ protein-coupled receptor to regulate its endocytosis through an Rac1-dependent mechanism. J Biol Chem. 2004;279:18981-9.

76. Tseng YH, Vicent D, Zhu J, et al. Regulation of growth and tumorigenicity of breast cancer cells by the low molecular weight GTPase Rad and nm23. Cancer Res. 2001;61:2071-9.

77. Srinivasan S, Traini M, Herbert B, et al. Proteomic analysis of a developmentally regulated secretory vesicle. Proteomics. 2001;1:1119-27.

78. Laurent O, Bruckert F, Adessi C, et al. In vitro reconstituted Dictyostelium discoideum early endosome fusion is regulated by Rab7 but proceeds in the absence of ATP-Mg2 + from the bulk solution. J Biol Chem. 1998;273:793-9.

79. Ma D, McCorkle JR, Kaetzel DM. The metastasis suppressor NM23-H1 possesses 3'-5' exonuclease activity. J Biol Chem. 2004;279:18073-84.

80. Novak M, Jarrett SG, McCorkle JR, et al. Multiple mechanisms underlie metastasis suppressor function of NM23-H1 in melanoma. Naunyn Schmiede Arch Pharmacol. 2011;384:433-8.

81. Bosnar MH, De Gunzburg J, Bago R, et al. Subcellular localization of A and B Nm23/NDPK subunits. Exp Cell Res. 2004;298:275-84. 
82. Bosnar MH, Bago R, Četković H. Subcellular localization of Nm23/NDPK A and B isoforms: a reflection of their biological function? Mol Cell Biochem. 2009;329:63-71.

83. Francois-Moutal L, Marcillat O, Granjon T. Structural comparison of highly similar nucleoside-diphosphate kinases: Molecular explanation of distinct membrane-binding behavior. Biochimie. 2014;105:110-8.

84. Francois-Moutal L, Ouberai MM, Maniti O, et al. Two-step membrane binding of NDPK-B induces membrane fluidity decrease and changes in lipid lateral organization and protein cluster formation. Langmuir ACS J Surf Colloids. 2016;32:12923-33.

85. Fancsalszky L, Monostori E, Farkas Z, et al. NDK-1, the homolog of NM23-H1/H2 regulates cell migration and apoptotic engulfment in C. elegans. PLoS ONE. 2014;9:e92687. 